\title{
Aportación de las personas mayores en los colegios intergeneracionales. La experiencia del proyecto ISCI
}

\section{Older adults' contribution in intergenerational schools. Experience from the ISCI project}

\author{
Mariano Sánchez*, Juan Sáez**, Margarita Campillo**, Pilar Díaz* \\ *Universidad de Granada, **Universidad de Murcia
}

\begin{abstract}
Resumen
ISCI es un proyecto de investigación cuyo objetivo es diseñar, pilotar y validar un modelo de colegio intergeneracional en tres centros de Educación Primaria situados en las comunidades de Andalucía y Región de Murcia. Inspirándose en la experiencia acumulada por The Intergenerational School, en Estados Unidos, ISCI se propone impulsar un modelo de colegio intergeneracional elaborado, innovador y basado en evidencias, que pueda recrearse en el contexto educativo español, atravesado, en parte, por el fracaso escolar y la incertidumbre. El artículo describe las tareas y las aportaciones de fondo de los mentores intergeneracionales del proyecto.

Palabras clave: colegio, intergeneracional, educación, personas mayores.
\end{abstract}

\begin{abstract}
ISCI is the name of a research project aimed at planning, piloting, and validating a model of intergenerational school. Three Primary Schools located in Andalucía and Murcia will be involved. The experience accumulated by The Intergenerational School, in United States, has inspired ISCI's approach. The project's goal is setting up and fostering an elaborate, innovative, and evidence-based model of intergenerational school which may be replicated in the Spanish educational context, one partially permeated by school failure and uncertainty. This paper presents both the tasks and the deeper contributions made by intergenerational mentors in the project.

Keywords: school, intergenerational, education, older people.
\end{abstract}

\section{Contextualización}

¿Qué hacen personas adultas y mayores en un Colegio de Primaria?, ¿por qué contar con ellas para mejorar la calidad de la enseñanza en las escuelas?, ¿de qué datos sobre la contribución de otras generaciones a la vida escolar, fruto de haber explorado experiencias sobre la colaboración de personas mayores en colegios centrados en la educación de niños y adolescentes?, ¿qué es lo que caracteriza el diseño y desarrollo de un colegio adjetivado de intergeneracional? Estas y otras preguntas se abordan en el proyecto ISCI (Intergenerational School - Colegio Intergeneracional), orientado a diseñar, validar y pilotar ese tipo de colegio, caracterizado por poner en relación, con perspectiva intergeneracional, a personas de diferentes generaciones.

El conocimiento de que se dispone hoy para dar respuesta a los anteriores interrogantes es más bien escaso, como ha puesto de manifiesto la literatura científica específica sobre espacios educativos intergeneracionales (Mannion, 2012). Con lo que sí contamos es con investigaciones en torno a los centros intergeneracionales, es decir, espacios en los que, de forma intencionada, se presta servicio conjuntamente a personas de distintas generaciones. Ya en un documento pionero dedicado a revisar los trabajos publicados sobre centros intergeneracionales y atención a la dependencia (Mañós, Pinazo, Sáez, \& Sáez, 2006) se describían algunos de los beneficios potenciales para los usuarios de estos centros. Por ejemplo, se ha demostrado que personas mayores que practican de forma regular el voluntariado con niños queman un $20 \%$ más de calorías a la semana, tienen menos caídas, utilizan menos el bastón y consiguen mejores resultados en pruebas de memoria en comparación con personas mayores no voluntarias.

En el caso de personas mayores dependientes, Xaverius \& Mathews (2003) pudieron concluir que un grupo de estas personas, diagnosticadas con demencia, tras interactuar durante 18 meses con 60 niños de 6-7 años procedentes de un centro escolar vecino, aumentaron sus niveles de implicación y expresividad en contraste con los mostrados en actividades no intergeneracionales. Otro investigadores concluyeron que los padres de niños que acudían a escuelas infantiles conectadas con centros de día para personas adultas admitían que sus hijos interactuaban de forma más positiva con ellos desde que los niños participaban en actividades intergeneracionales (Liou, Mendes, \& Jarrott, 2011). Asimismo, Femia, Zarit, Blair, Jarrott y Bruno (2008) encontraron que los niños que habían acudido a una escuela infantil con programación intergeneracional lograban puntuaciones más altas en aceptación social de las personas mayores que otro 
grupo de niños inscritos en una escuela infantil tradicional.

En conclusión, contamos con algunas evidencias del potencial de cambio que tiene un centro intergeneracional. Sobre estas evidencias habría que profundizar. Sin embargo, nos falta más estudio acerca de qué sucede cuando uno de esos centros es un colegio dedicado a la escolarización obligatoria.

\section{El predominio de la mentorización}

En colegios de muchos países se realizan actividades denominadas intergeneracionales. $\mathrm{Y}$ cuando de las actividades aisladas se pasa a los programas, podríamos decir que, hasta ahora, en el ámbito escolar, el modelo de programa intergeneracional más extendido y en torno al cual disponemos de más conocimiento científico-técnico es el de la mentorización. Si se lleva a cabo de forma adecuada, una relación de mentorización positiva y duradera entre una persona adulta y un niño o un adolescente puede tener un impacto muy positivo sobre la autoestima, las competencias sociales y académicas, y la motivación e implicación educativas del menor (Komosa-Hawkins, 2009). También se ha comprobado que estos alumnos, gracias al apoyo de un mentor, se sienten más conectados con su centro escolar $\mathrm{y}$, por ello, disminuyen la probabilidad de fracaso escolar (Portwood \& Ayers, 2005) y mejoran los resultados académicos durante la educación obligatoria.

En el caso particular de los alumnos de Educación Primaria -en quienes se centra el proyecto ISCI- las evidencias científicas más sólidas producidas hasta la fecha con relación a la mentorización intergeneracional escolar provienen de las evaluaciones del proyecto norteamericano "Experience Corps", en el cual más de 2.000 tutores voluntarios de 55 o más años de edad ayudan a mejorar su competencia lectora a unos 20.000 alumnos de Educación Primaria. Una de las evaluaciones de más calado de "Experience Corps" realizadas hasta la fecha estuvo a cargo de Morrow-Howell, Jonson-Reid, McCrary, Lee, \& Spitznagel (2009). Utilizaron metodología experimental totalmente aleatorizada con alumnos de 6-8 años de 23 escuelas norteamericanas y demostraron la significatividad estadística del aumento de competencia lectora en esos niños y niñas -todos ellos con niveles bajos de competencia lectora al inicio de la intervención- tras haber participado en un mínimo de 35 sesiones de trabajo con tutores mayores. En cuanto al impacto del programa sobre los mentores, esta misma evaluación constató un descenso significativo de la depresión, un aumento de la actividad y un crecimiento del círculo de amistades en la mayoría de esos tutores.

\section{El modelo de Colegio Intergeneracional que inspira el proyecto ISCI}

La dimensión innovadora del proyecto ISCI consiste en diseñar, pilotar y validar un modelo de colegio intergeneracional aplicable a la etapa de Educación Primaria, viable y sostenible, capaz de mejorar las tasas de éxito escolar en el contexto geográfico de dos Comunidades Autónomas españolas -la Comunidad Autónoma de Andalucía y la Región de Murcia- en una doble dirección: la mejora de las competencias de los alumnos y el aumento de la calidad de vida de las personas adultas y mayores participantes.

Hasta la fecha contamos en España con numerosos ejemplos de proyectos intergeneracionales llevados a cabo en centros escolares pero no disponemos aún de un centro escolar que podamos decir que responde por completo a la denominación y filosofía de un "colegio intergeneracional". ¿Qué hay detrás de este concepto? La incorporación de la intergeneracionalidad y el enfoque del ciclo vital como principios rectores transversales a toda la labor educativa llevada a cabo en el colegio. En términos educativos esto implica la puesta en práctica, desde edades tempranas, del aprendizaje a lo largo de toda la vida mediante la facilitación de interacciones planificadas, científicamente orientadas, cotidianas y significativas entre personas de distintas generaciones que compartan espacios, tiempos y culturas en el centro educativo.

Ahora bien, hace falta proporcionarles a nuestros centros escolare un modelo validado de educación intergeneracional. $\mathrm{Y}$ eso es precisamente lo que nos hemos propuesto hacer al fundamentar nuestro proyecto de investigación en el mejor y más reconocido ejemplo de colegio intergeneracional que creemos que existe en este momento en el contexto internacional: The Intergenerational School (en adelante, TIS), ubicado en la ciudad norteamericana de Cleveland (Ohio). ¿Qué es TIS? Es una escuela concertada gratuita, para niños de 5 a 14 años, fundada en el año 2000, que, en consecuencia con su decisión de trabajar desde la perspectiva del ciclo vital, reúne dos características que la hacen distinta a cualquiera otra de su entorno y le confieren su naturaleza intergeneracional:

- Aulas multi-grado: En lugar de asignar a los alumnos a cada aula de modo que, en cada curso, todos tengan la misma edad cronológica, TIS trabaja con aulas multi-etarias organizadas en tres etapas de desarrollo.

- Personas adultas como tutores-mentores: Unas 70 personas adultas -algunas de ellas afectadas de demencia- participan, de forma cotidiana, planificada y supervisada, en las tareas educativas apoyando a los maestros. Por ejemplo, actúan como tutores voluntarios ayudando a los niños a mejorar su competencia lectora y matemática.

\section{TIS, orientada a la mejora escolar de los niños y adolescentes}

Algunas conclusiones obtenidas de estudios sobre TIS permiten una aproximación más precisa a lo que es un colegio intergeneracional. Así, según datos oficiales del Departamento de Educación del Estado de Ohio, en el curso académico 2005-06, y tras evaluación mediante pruebas estandarizadas, el $100 \%$ de los alumnos de 8-9 años de TIS - frente a un 50,7\% de los niños matriculados en el distrito de Cleveland- eran capaces de leer a un nivel óptimo. Poco más tarde, en 2009, los resultados de una nueva evaluación estandarizada, realizada por ese mismo organismo, concluyeron que el $100 \%$ de los alumnos de 11 años de TIS habían alcanzado los niveles más altos de lectura exigibles a su edad -este porcentaje fue del $82 \%$ para el conjunto de 
los centros escolares de Ohio. En el caso de la materia de matemáticas, el $92,3 \%$ de los estudiantes de TIS demostraron competencias propias de los niveles superiores -en contraste con un 75\% de los alumnos de la misma edad en el Estado de Ohio.

Más datos. En 2013, TIS fue reconocido como "High-Performing School of Honor", distinción que tan solo lograron 37 centros en el Estado de Ohio. Esto significa que durante los cinco años anteriores TIS había conseguido, entre otros méritos, que el nivel general de competencia de sus alumnos -medido a través pruebas oficiales estandarizadas- fuese, como mínimo y por término medio, superior a 90, en una escala 1-100. Un año más tarde, en 2014, la Fundación norteamericana Eisner concedió a TIS el premio al mejor programa intergeneracional en Estados Unidos.

Por otro lado, en la literatura especializada sobre centros escolares intergeneracionales, TIS aparece como uno de los pocos lugares en los que se está investigando in situ al respecto. El profesor Whitehouse, co-fundador de TIS, planteó en su momento que la puesta en marcha de TIS era la respuesta a una doble y creciente necesidad: crear nuevas oportunidades de aprendizaje para diversos grupos etarios a lo largo del ciclo vital y ampliar el apoyo a familias y comunidades (Whitehouse, Bendezu, FallCreek, \& Whitehouse, 2000).

\section{TIS, orientado a la mejora de niños y adolescentes}

Ya hemos visto algo acerca del impacto académico de TIS en sus alumnos. ¿Qué potencial tiene este tipo de centro sobre la mejora del bienestar entre las personas adultas y mayores? Por ejemplo, sabemos que un grupo de personas mayores con demencia media y moderada, participantes en encuentros intergeneracionales semanales realizados en TIS y planificados con niños de 5-14 años, mejoraron su calidad de vida - con una significativa disminución de estrés- medida a través de cinco variables psicométricas (funcionamiento cognitivo, estrés, depresión, sensación de tener un propósito en la vida y sentimiento de utilidad) (George $\&$ Singer, 2011). A través de observación participante y de entrevistas estructuradas y no estructuradas a cuidadores, familiares y personal, esa intervención demostró mejoría en la salud, en la sensación de utilidad y en las relaciones entre dichas personas mayores.

Más recientemente el profesor Whitehouse (2013) ha hecho un balance sintético de las claves del éxito de TIS: construcción social, excelencia en la educación, aprendizaje experiencial mediante el servicio a lo largo de toda la vida, y participación en la vida política y social. TIS está intentando que otras organizaciones se transformen en lo que este colegio ya es: una entidad de aprendizaje multi-etario, en la que personas adultas de diversas edades colaboran porque las experiencias educativas que TIS pone a su alcance les resultan significativas. Este contexto de implementación y crecimiento del modelo de TIS nos ofrece una oportunidad idónea para la realización del proyecto ISCI en España. De ahí que en el curso académico 2016/17 hayamos comenzado a pilotar una versión adaptada del modelo de TIS en tres colegios: el Centro de Educación Infantil y Primaria (CEIP) Ramón Gaya, situado en la localidad murciana de Santomera, y los CEIP Federico García Lorca y Gallego Burín, de Granada capital.

\section{Lo aprendido hasta la fecha}

Después de los seis primeros meses de experimentación del modelo hemos acumulado experiencia suficiente para plantear el siguiente paso: la implementación durante un curso académico completo. Por ahora solo estamos en disposición de extraer conclusiones muy preliminares y algunas orientaciones para la mejora continua del proceso. Pasamos a hablar de ello con el fin de que nuestro trabajo pueda servir de inspiración a otros investigadores y centros escolares.

La primera lección aprendida es que existe una profunda diferencia entre el trabajo intergeneracional basado en actividades aisladas y pensadas como elementos añadidos al día a día del centro escolar -por ejemplo, la organización de una visita puntual de los alumnos a un centro residencial de personas mayores del entorno- y la introducción progresiva de personas adultas y mayores en el flujo normal y planificado del colegio. Nuestro modelo ha apostado por esto último, e incluso ha dado la vuelta a la lógica más habitual utilizada a la hora de realizar actividades intergeneracionales aisladas: nuestros mentores se han sumado, cuando la comunidad escolar les ha invitado, a las iniciativas que, en cualquier caso, los tres colegios participantes en el proyecto habrían llevado a cabo, con independencia de poder contar con el apoyo de dichos mentores. No es lo mismo introducir un evento intergeneracional en un momento dado que -por poner un ejemplo- plantearse cómo introducir la intergeneracionalidad en una semana cultural que uno de nuestros centros escolares viene celebrando hace años. En este último caso la intergeneracionalidad se incorpora -como perspectiva de mejora- al flujo de vida del colegio aportando su potencial de transformación. Dicho de otro modo, la cultura del centro escolar -sus hábitos, rutinas, símbolos, prácticas, ritmos, lenguajes, etc.- acoge y le abre hueco al vector intergeneracional, que la penetra lentamente y de modo transversal. Quizá haya sido esta manera de proceder la que ha hecho que nuestros mentores admitan haber conseguido sentirse, tras seis meses de participación a razón de una media de dos horas semanales, parte del centro escolar. Aquí surge el tema del reconocimiento: tras este periodo inicial los mentores se sienten reconocidos -se sabe quiénes son y lo que hacen- $\mathrm{y}$, a su vez, la comunidad educativa reconoce el proceso en el que se ha embarcado gracias a este proyecto.

Por otro lado, debemos subrayar la relevancia que ha tenido que la colaboración de los mentores haya sido regular, planificada, continuada y, sobre todo, orientada por los criterios que han marcado las maestras de primer y segundo curso de Educación Primaria. La apertura de un espacio para la mentorización intergeneracional en cada aula-grupo solo ha sido posible por la mediación de las maestras, cuyo papel en el proyecto se ha caracterizado por lo siguiente: (i) acogida de los mentores, (ii) mediación en el proceso de introducción 
del mentor como parte del grupo, (iii) decisión sobre las tareas a realizar -siempre relevantes para los objetivos marcados por el currículum en la etapa educativa concreta de intervención-, (iv) socialización del mentor en las reglas de funcionamiento $\mathrm{y}$ en la cultura relacional del aula, (v) transmisión al mentor de información relevante para la mayor eficacia de la mentorización, (vi) orientación al mentor acerca del modo más adecuado de llevar a cabo las tareas encomendadas, (vii) seguimiento de las horas de trabajo niño-mentor, y (viii) aportación de comentarios valorativos en los momentos de evaluación de la marcha del proyecto. Una conclusión emerge con fuerza: el modelo que estamos implementando coloca a las maestras -en nuestro caso, todas ellas son mujeres- en el centro, como clave en torno a la cual pivota el día a día del proyecto en el centro escolar. Este trasvase del control del proyecto al espacio no solo del colegio en general sino del aula en particular es una característica claramente diferenciadora de lo que estamos haciendo por contraste con el procedimiento en el que existe una entidad externa con la que el centro escolar colabora en la puesta en marcha de una actividad intergeneracional. En el modelo del proyecto ISCI el locus de control está en el centro escolar dado el carácter eminentemente educativo de lo que estamos haciendo.

¿Qué tareas han llevado a cabo nuestros mentores? La fundamental ha sido la de acompañamiento a la lectura. Se han llevado a cabo sesiones semanales de unos 15 minutos de duración con todos los niños y niñas, habitualmente en grupos pequeños (2-3 alumnos más un mentor). Estas sesiones han permitido no solo practicar la lectura sino todo lo que lleva asociado: comunicación oral, diálogo, relato de experiencias, conocimiento mutuo, explicaciones sobre lo leído, y, lo que es más importante, creación de confianza y de sentido de relación. Del mismo modo los mentores han trabajado también en tareas básicas propias del currículo de matemáticas: operaciones de cálculo, familiarización con números y cifras, resolución de problemas y fijación de estrategias de uso del razonamiento matemático en situaciones de la vida cotidiana. La atención a estas dos áreas curriculares ha sido prioritaria.

Sin embargo, los mentores han hecho mucho más. Por ejemplo, (i) visualizar, en el día a día y mediante su labor en los centros escolares piloto, ejemplos de compromiso cívico por parte de personas adultas y mayores, (ii) erosionar estereotipos negativos sobre el envejecimiento, (iii) ampliar el perfil de personas implicadas en las comunidades educativas de los tres colegios, (iv) insuflar nuevos lenguajes, narrativas, símbolos y formas de hacer, nueva vida, en definitiva, en los espacios de educación formal, y (v) contribuir al optimismo con respecto a las posibilidades reales de mejora de los aprendizajes demostrando que la marcha de la educación es relevante para la comunidad externa al colegio. Ahora bien, si ahondamos un poco más nos encontramos con algunas contribuciones que creemos que supondrán transformaciones de calado en el habitus educativo de los colegios; nos referimos al impulso de una cultura de las relaciones, a la ampliación del sentido del tiempo en el contexto escolar y a la deconstrucción del centrismo de las edades en la articulación de los procesos educativos.

¿Por qué la presencia de personas adultas y mayores externas a un colegio y ubicadas como mentores puede alterar la cultura relacional? Pues porque estos mentores acuden al colegio por el placer de participar e introducen en el día a día momentos de relaciones no marcadas por la obligación, no por el rol o el estatus, sino por el deseo de encontrarse. En un colegio intergeneracional como el que vislumbramos aumentarán las oportunidades de relacionarse sin imperativos, sin forzamiento, dejando más paso a las emociones, a lo instantáneo, concreto e inesperado. Pensamos que este nuevo aire relacional puede oxigenar seriamente contextos escolares en los que sobreabundan los preceptos, las normativas, las obligaciones y lo estructurado.

En cuanto al tema del sentido del tiempo, de la comprensión de la temporalización de nuestras vidas, la cosa parece clara: unos niños y niñas que entrar en contacto cotidiano con más personas de muchas más edades pueden acceder a una socialización distinta al respecto de la duración de la vida humana y de la experiencia de las distintas maneras de entender el tiempo. Un ejemplo como el siguiente aclarará mejor lo que decimos; si un mensaje habitual que escuchamos en un aula escolar es "Niños, no perdáis el tiempo", en una conversación niño-mentor puede surgir una reflexión como esta: "Al final, tras muchos años de ir corriendo por la vida me doy cuenta de que he llegado al mismo sitio; de poco me ha servido correr tanto". Aquí vemos dos formas alternativas de aludir a la temporalidad humana. Parece razonable suponer que si aumenta el repertorio de edades y experiencias temporales presentes en un colegio, también lo haga la alfabetización sobre el entendimiento de los tiempos.

Por último, y a medida que una interacción intergeneracional adecuadamente guiada se lleva a cabo, podemos esperar que sean las relaciones, las experiencias y el sentido de pertenencia lo que vaya ganando terreno frente a la construcción edad-curso. ¿Por qué? Porque al mismo tiempo que la presencia de los mentores quiebra la uniformidad etaria de las aulas, el eje de trabajo intergeneracional invita poco a poco al centro escolar a cruzar alumnos de distintas edades en tareas colaborativas. Con ello, la fuerza de la edad como marcador de quién eres y qué haces en el colegio se pone en cuestión.

\section{Reflexión final}

Esperamos haber ayudado a cuestionar que aportar por un colegio intergeneracional tiene que ver con la mera realización de actividades en las que participen personas de distintas generaciones. No. Sobre todo tiene que ver con la transformación de las prácticas educativas mediante la potenciación de la cultural relacional -mediante el anudamiento de las generaciones-, el enriquecimiento de los sentidos de la temporalidad -más allá de la tradicional cronología etaria-, la visión del aprendizaje como proceso factible 
e indispensable a lo largo de todo el ciclo vital, y la ejemplaridad del compromiso cívico y desinteresado en el bien común. Y todo esto solo es posible si asumimos que un centro de Educación Primaria también es un lugar no solo "para todas las edades"-como parece haberse puesto de moda afirmar acerca de ciudades, empresas, sociedades, etc.- sino "para deshacer y recomponer las edades" a base de oportunidades de relaciones y vínculos descomprometidos entre personas que se perciben en trayectoria vital pero ocupando distintas posiciones generacionales.

\section{Agradecimientos}

El Proyecto ISCI está contando con la valiosa implicación de las autoridades educativas de la Región de Murcia y de la Junta de Andalucía, sin las cuales el pilotaje no sería posible; por ello, expresamos nuestro agradecimiento a los equipos técnicos de las respectivas Consejerías responsables de educación. Por supuesto, el agradecimiento se extiende a nuestros mentores, a los equipos directivos $\mathrm{y}$ docentes de los seis CEIP implicados, y a los alumnos y las familias de esos centros escolares.

El proyecto ISCI está financiado por el Ministerio de Economía y Competitividad del Gobierno de España.

\section{Referencias}

Femia, E.E., Zarit, S.H., Clancy, B., Jarrott, S.E., \& Bruno, K. (2008). Intergenerational preschool experiences and the young child: Potential benefits to development. Early Childhood Research Quarterly, 23, 272-287. doi:10.1016/j.ecresq.2007.05.001

George, D.R. \& Singer, M.E. (2011). Intergenerational Volunteering and Quality of Life for Persons With Mild to Moderate Dementia: Results From a 5-Month Intervention Study in the United States. American Journal of Geriatric Psychiatry, 19(4), 392-396. doi: 10.1097/JGP.0b013e3181f17f20

Komosa-Hawkins, K. (2009). Best Practices in School-Based Mentoring Programs for Adolescents. Child \& Youth Services, 31(3), 121-137. doi: 10.1080/0145935X.2009.524477

Liou, C.L., Mendes, A.B., \& Jarrott, S.E. (2011). Schools for all ages: How intergenerational programs nurture the youngest-young and oldest-old. Aging Today, xxxii(5), 9-12.

Mannion, G. (2012). Intergenerational Education: The significance of 'reciprocity' and 'place'. Research project report. Recuperado de www.scotip.org.uk

Mañós, F., Pinazo, S., Sáez, J. y Sánchez, M. (2006). Los centros intergeneracionales en la atención a la dependencia. Fundamentos, funcionamiento $\mathrm{y}$ resultados. Madrid: IMSERSO. Recuperado de htttp://www.envejecimiento.csic.es

Morrow-Howell, N., Jonson-Reid, M., McCrary, S., Lee, Y., \& Spitznagel, E. (2009). Evaluation of Experience Corps: Student Reading Outcomes. St. Louis, MO: Washington University, Center for Social Development.

Portwood, S. G. \& Ayers, P. M. (2005). Schools. In D. L. DuBois \& M. J. Karcher (Eds.), Handbook of
Youth Mentoring (pp. 336-347). Thousand Oaks, CA: Sage.

Xaverius, P.K. \& Mathews, R.M. (2003). Evaluating the Impact of Intergenerational Activities on Elders' Engagement and Expressiveness Levels in Two Settings. Journal of Intergenerational Relationships, 1(4), 53-69.

Whitehouse, P. (2013). The Challenges of Cognitive Aging: Integrating Approaches from Neuroscience to Intergenerational Relationships. Journal of Intergenerational Relationships, 11(2), 105-117. doi: 10.1080/15350770.2013.782740

Whitehouse, P.J., Bendezu, E., FallCreek, S., \& Whitehouse, C. (2000). Intergenerational community schools: a new practice for a new time. Educational Gerontology, 26, 761-770. 